\title{
A novel COVID-19 and its effects on cardiovascular disease
}

\author{
Arumugam Paramasivam ${ }^{1}$ - Jayaseelan Vijayashree Priyadharsini ${ }^{1} \cdot$ Subramanian Raghunandhakumar $^{1}$. \\ Perumal Elumalai ${ }^{2}$
}

Received: 8 April 2020 / Revised: 10 April 2020 / Accepted: 12 April 2020 / Published online: 30 April 2020

(C) The Japanese Society of Hypertension 2020

Coronavirus disease (COVID-19) is a novel infectious disease caused by a newly discovered severe acute respiratory syndrome coronavirus-2 (SARS-CoV-2) [1, 2]. COVID-19 spread rapidly from China, reaching a pandemic and putting the world on alert. SARS-CoV-2 has been reported in 211 countries, areas or territories, and $>1,200,000$ cases have been confirmed, with an estimated mortality risk of $\sim 5.5 \%$ (https://www.who.int/emergencies/diseases/novel-corona virus-2019). In India, 5000 patients were confirmed with SARS-CoV-2, with an estimated mortality risk of $\sim 2.5 \%$ and an escalating incidence rate increasing daily. Unfortunately, there are no specific vaccines or treatments for COVID-19 at this time, and current management includes travel restrictions, patient isolation, and supportive medical care. However, scientists are endeavoring to discover drugs and vaccines for COVID-19, but a better understanding of the underlying pathobiology is required.

More recent research has suggested that older people and people with chronic health conditions, including cardiovascular disease (CVD), are at higher risk for mortality and morbidity related to COVID-19 than the average population [2]. In a case series of 21 patients with COVID-19, congestive heart failure was the second most common baseline comorbidity (42.9\%) [3]. A new study reported that COVID-19 can cause heart injury, even in people without underlying heart issues [2]. However, while the clinical manifestations of COVID-19 are dominated by respiratory symptoms, cardiovascular involvement can occur through several other mechanisms [4]. Acute cardiac injury is the most frequently

Arumugam Paramasivam

paramasivam0103@gmail.com

1 BRULAC-DRC, Saveetha Dental College \& Hospital, Saveetha Institute of Medical and Technical Sciences [SIMATS], Saveetha University, Chennai, India

2 Central Research Laboratory, Meenakshi Medical College Hospital \& Research Institute, Meenakshi Academy of Higher Education and Research (MAHER), Chennai, India reported cardiovascular abnormality in COVID-19, and it occurs in $\sim 8-12 \%$ of all patients [5]. The presence of underlying CVD and/or development of acute cardiac injury might confer an increased risk of death (Table 1).

A recent study reported the importance of cardiac injury in patients with COVID-19; in these patients, $10.6 \%$ had coronary heart disease, $4.1 \%$ had heart failure, and $5.3 \%$ had cerebrovascular disease. Approximately $20 \%$ of patients had cardiac injury. A similar study demonstrated factors associated with outcomes in 187 patients with COVID-19. They showed that $35 \%$ had underlying CVD (hypertension, coronary heart disease, or cardiomyopathy), and $28 \%$ showed evidence of acute myocardial injury (defined as elevated troponin T). Furthermore, mortality was significantly higher in individuals with high troponin $\mathrm{T}$ levels than in those with normal troponin $\mathrm{T}$ levels. In addition, patients with high troponin $\mathrm{T}$ levels were older and had more comorbidities, including hypertension, coronary heart disease, cardiomyopathy, and chronic kidney disease [2].

Another study showed that the incidence of acute heart failure was 23\% (44 out of 191 patients with COVID-19) and that multiple precipitating etiologies, including acute coronary syndrome, cardiac arrhythmias, stress-induced cardiomyopathy, and fulminant myocarditis, might result in acute heart failure or cardiogenic shock in patients with COVID-19 [6]. Lactate dehydrogenase (LDH), creatine kinase (CK) and their isoenzymes as well as the protein troponin I (TnI), which exhibited better cardiac specificity, are linked with injury of the heart muscle. Chen et al. reported that among the 99 confirmed COVID-19 patients, $13(13 \%)$ presented elevated CK, and $75(76 \%)$ showed elevated LDH [7]. Wang et al. revealed the clinical characteristics of 138 hospitalized COVID-19 patients, showing elevated $\mathrm{TnI}$ in $10(7.2 \%)$, whereas $23(16.7 \%)$ had arrhythmia [8]. In addition, Guan et al. demonstrated that $13.7 \%$ of COVID-19 patients showed elevated CK levels, and $37.2 \%$ showed increased LDH levels [9]. Although SARS-CoV-2 potentially invades alveolar epithelial cells, resulting in an acute systemic inflammatory response $[4,6]$, 
Table 1 Cardiovascular complications in COVID-19

\begin{tabular}{lll}
\hline Clinical manifestation & Incidence & Reference \\
\hline $\begin{array}{l}\text { Cardiovascular abnormality (increased cardiac } \\
\text { troponin I) }\end{array}$ & $8-12 \%$ & {$[5]$} \\
Heart failure & $52 \%$ in those who died and 12\% in those & {$[6]$} \\
& $\begin{array}{l}\text { who recovered } \\
\text { Acute cardiac injury }\end{array}$ & $59 \%$ in those who died and 1\% in those \\
& who recovered
\end{tabular}

the exact etiology and mechanisms of elevation of cardiac biomarkers among patients with COVID-19 have not yet been reported. The identification of angiotensin-converting enzyme 2 (ACE2) as a functional receptor for coronaviruses, including SARS-CoV and SARS-CoV-2, provides clues about their indirect association with cardiovascular pathology. The ACE2 receptor, the binding point for SARS-CoV-2, is abundantly found in myocytes. Therefore, myocyte damage from a direct viral attack could very well be the predominant mechanism.

ACE2 is a membrane-bound aminopeptidase that is highly expressed in the heart and lungs, and has a vital role in the cardiovascular and immune systems in normal health as well as in various disease conditions [1, 10]. ACE2 is also involved in heart function and the development of hypertension and diabetes mellitus. The binding of SARS-CoV-2 to ACE2 can result in alteration of ACE2 signaling pathways, leading to acute myocardial and lung injury. COVID-19 is more severe in patients with CVD, which might be associated with increased secretion of ACE2 in these patients compared with healthy individuals $[1,10]$.

In conclusion, there seems to be a unique interplay between SARS-CoV-2 and CVDs. Although COVID-19 is predominantly a respiratory illness, a large number of patients with COVID-19 present with preexisting CVD or develop new-onset cardiac dysfunction during the course of the illness. Therefore, understanding the CVD caused by SARS-CoV-2 and the underlying mechanisms is of the greatest importance, and during treatment for COVID-19, careful attention should be given to cardiovascular protection.

\section{Compliance with ethical standards}

Conflict of interest The authors declare that they have no conflicts of interest.
Publisher's note Springer Nature remains neutral with regard to jurisdictional claims in published maps and institutional affiliations.

\section{References}

1. Li G, Hu R, Zhang X. Antihypertensive treatment with ACEI/ ARB of patients with COVID-19 complicated by hypertension. Hypertens Res. 2020. https://doi.org/10.1038/s41440-020-0433-1.

2. Madjid M, Safavi-Naeini P, Solomon SD, Vardeny O. Potential effects of coronaviruses on the cardiovascular system: a review. JAMA Cardiol. 2020. https://doi.org/10.1001/jamacardio.2020. 1286.

3. Arentz M, Yim E, Klaff L, Lokhandwala S, Riedo FX, Chong M, et al. Characteristics and outcomes of 21 critically ill patients with COVID-19 in Washington State. JAMA. 2020. https://doi.org/10. 1001/jama.2020.4326.

4. Huang C, Wang Y, Li X, Ren L, Zhao J, Hu Y, et al. Clinical features of patients infected with 2019 novel coronavirus in Wuhan, China. Lancet. 2020;395:497-506.

5. Lippi G, Plebani M. Laboratory abnormalities in patients with COVID-2019 infection. Clin Chem Lab Med. 2020. https://doi. org/10.1515/cclm-2020-0198.

6. Zhou F, Yu T, Du R, Fan G, Liu Y, Liu Z, et al. Clinical course and risk factors for mortality of adult inpatients with COVID-19 in Wuhan, China: a retrospective cohort study. Lancet. 2020. https:// doi.org/10.1016/S0140-6736(20)30566-3.

7. Chen N, Zhou M, Dong X, Qu J, Gong F, Han Y, et al. Epidemiological and clinical characteristics of 99 cases of 2019 novel coronavirus pneumonia in Wuhan, China: a descriptive study. Lancet. 2020;395:507-13.

8. Wang D, Hu B, Hu C, Zhu F, Liu X, Zhang J, et al. Clinical characteristics of 138 hospitalized patients with 2019 novel coronavirus-infected pneumonia in Wuhan, China. JAMA. 2020; e201585:E1-E9.

9. Guan WJ, Ni ZY, Hu Y, Liang WH, Ou CQ, He JX, et al. China Medical Treatment Expert Group for Covid-19. Clinical characteristics of coronavirus disease 2019 in China. N Engl J Med. 2020. https://doi.org/10.1056/NEJMoa2002032.

10. Letko M, Marzi A, Munster V. Functional assessment of cell entry and receptor usage for SARS-CoV-2 and other lineage B betacoronaviruses. Nat Microbiol. 2020;5:562-569. 